\title{
Random line tessellations of the plane: statistical properties of many-sided cells
}

\author{
H. J. Hilhorst ${ }^{1}$ and P. Calka ${ }^{2}$ \\ ${ }^{1}$ Laboratoire de Physique Théorique, Bâtiment 210 \\ Univ Paris-Sud and CNRS, 91405 Orsay Cedex, France \\ ${ }^{2}$ Laboratoire MAP5, Université Paris Descartes, 45, rue des Saints-Pères \\ 75270 Paris Cedex 06
}

October 26, 2018

\begin{abstract}
We consider a family of random line tessellations of the Euclidean plane introduced in a much more formal context by Hug and Schneider [Geom. Funct. Anal. 17, 156 (2007)] and described by a parameter $\alpha \geq 1$. For $\alpha=1$ the zero-cell (that is, the cell containing the origin) coincides with the Crofton cell of a Poisson line tessellation, and for $\alpha=2$ it coincides with the typical Poisson-Voronoi cell. Let $p_{n}(\alpha)$ be the probability for the zero-cell to have $n$ sides. By the methods of statistical mechanics we construct the asymptotic expansion of $\log p_{n}(\alpha)$ up to terms that vanish as $n \rightarrow \infty$. In the large- $n$ limit the cell is shown to become circular. The circle is centered at the origin when $\alpha>1$, but gets delocalized for the Crofton cell, $\alpha=1$, which is a singular point of the parameter range. The large- $n$ expansion of $\log p_{n}(1)$ is therefore different from that of the general case and we show how to carry it out. As a corollary we obtain the analogous expansion for the typical $n$-sided cell of a Poisson line tessellation.
\end{abstract}

Keywords: random line tessellations, Crofton cell, exact results

LPT Orsay 08-17 


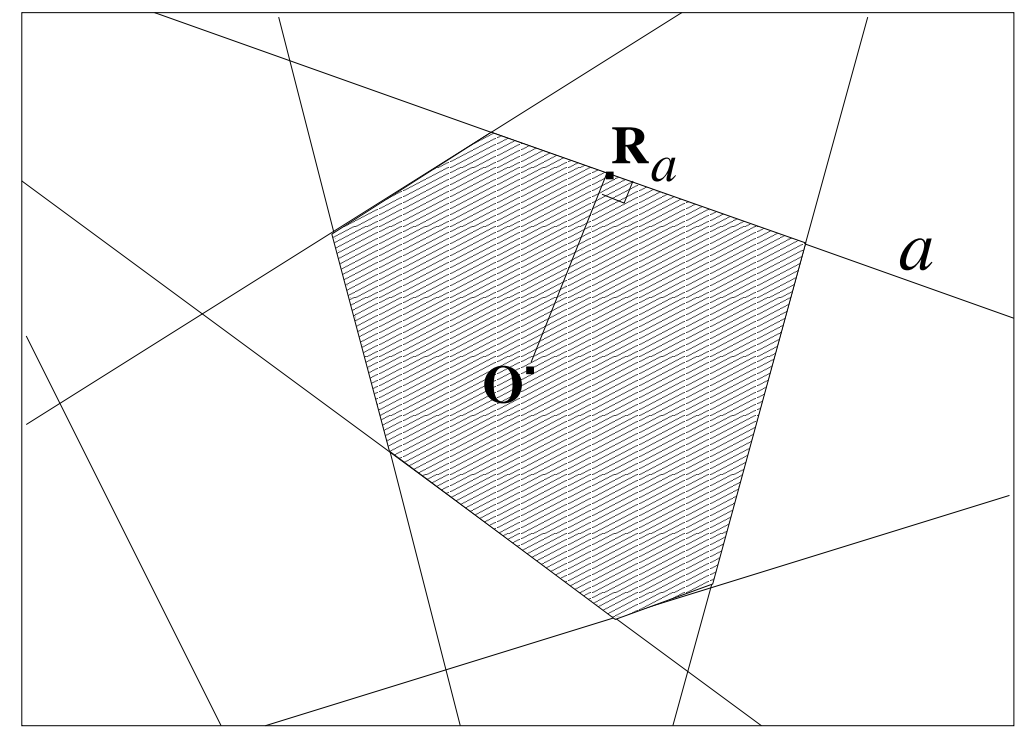

Figure 1: Example of a line tessellation of the plane. The projection of the origin onto line $a$ is denoted $\mathbf{R}_{a}$; this projected point fully determines $a$. The cell surrounding the origin (the 'zero-cell') has been shaded.

\section{Introduction}

\subsection{Poisson-Voronoi and Poisson line tessellations}

A Voronoi tessellation of $\mathbb{R}^{2}$ is a partitioning of the plane into cells constructed around 'point particles' in such a way that each point of space is in the cell of the particle to which it is closest. When the point particle distribution is uniformly random, (or, in mathematical terms, when it corresponds to a homogeneous Poisson point process), the resulting partition is said to be a Poisson-Voronoi tessellation. It is one of the simplest mathematical models of naturally occurring planar cellular structures. Because of the great variety of their applications, the statistics of Poisson-Voronoi cells has been studied in many different areas of science. References may be found in the encyclopedic review by Okabe et al. [1].

A different way of partitioning the plane into cells is by means of intersecting straight lines, as in figure 1. When these have a uniform distribution (or, in mathematical terms, when they correspond to a homogeneous Poisson line process), we refer to the partition as a Poisson line tessellation. The Poisson-Voronoi tessellation and the Poisson line tessellation are both statistically invariant under translations and rotations in the plane. For both, the cells are convex polygons.

An early application of the Poisson line tessellation occurs in work by 
Goudsmit [2], carried out at the suggestion of Niels Bohr. The question in that paper arose from cloud chamber experiments: when three lines seemingly originate from the same point, then what is the probability that they do not result from the same event? Hence the problem became to calculate the probability for three independent lines to nearly pass through the same point, or, put differently, for a typical triangular cell to have an area less than $A$ in the limit of very small $A$. Poisson line tessellations have since interested mathematicians with important contributions due to, in particular, Miles [3, 4, 5], Matheron [6], Kovalenko [7, 8], Goldman [9], and Hug, Reitzner and Schneider [10].

The cell that contains the origin is generally called the 'zero-cell'. In the Poisson line tessellation it carries the special name of 'Crofton cell', in reference to Crofton's formula in integral geometry [11]. Since the origin falls in a cell of area $A$ with a probability proportional to $A$, the Crofton cell is not typical but more likely larger-than-typical. In mathematical terms, the density of the typical cell differs from that of the Crofton cell by a factor $A /\langle A\rangle^{\text {typ }}$, where $\langle A\rangle^{\text {typ }}$ is the average typical-cell area. We will distinguish quantities pertaining to the typical cell of a Poisson line tessellation (as opposed to the Crofton cell) by an extra superscript 'typ'.

\subsection{Cell sidedness}

The cell property most studied is the sidedness probability $p_{n}$, that is, the probability for the cell to have $n$ sides. Other quantities of interest have included the averages, moments, and correlations of $n$, the cell area and the cell perimeter, as well as the distribution of the angles between the perimeter segments. The statistical properties of an $n$-sided cell may be expressed analytically as $2 n$-fold integrals on the planar coordinates of the point particles (for the Voronoi tessellation) or of the lines (for the line tessellation) defining that cell. However, only few of these integrals can be evaluated exactly. In particular the calculation of the fraction $p_{n}$ has so far been impossible for general $n$, whether for the Poisson-Voronoi or for the Poisson line tessellation.

We briefly recall some known results. In a Poisson line tessellation the typical cell has an average number of sides $\langle n\rangle^{\text {typ }}=4$. The sidedness $p_{n}^{\text {typ }}$ peaks at $n=4$. Miles [3] obtained the exact value $p_{3}^{\text {typ }}=2-\pi^{2} / 6=0.35506 \ldots$ and Tanner [12] showed that $p_{4}^{\text {typ }}=\pi^{2} \log 2-\frac{1}{3}-\frac{7}{36} \pi^{2}-\frac{7}{2} \zeta(3)=0.38146 \ldots$. Numerical values for $p_{n}^{\text {typ }}$ based on Monte Carlo simulation were given by Crain and Miles [13], by George [14, and recently by Michel and Paroux [15] (who compare their results to the earlier ones), for sidednesses not exceeding $n=12$.

For the Crofton cell, by contrast, Matheron [6] showed that the average sidedness is $\langle n\rangle=\pi^{2} / 2=4.9348 \ldots$. The distribution $p_{n}$ peaks at $n=5$. Miles [5] obtained the only known exact result, namely $p_{3}=(25-$ 
$36 \log 2) \pi^{2} / 6=0.076820 \ldots$ Numerical values for $p_{n}$ were given by Calka [16] for $n=3,4, \ldots, 9$ and by Michel and Paroux [15] for $n=3,4, \ldots, 11$.

The Poisson-Voronoi cell has an average sidedness of $\langle n\rangle=6$. Its sidedness distribution $p_{n}$ peaks at $n=6$. Although no exact results are known for any of the $p_{n}$, it has recently been possible [17, 18, 19] to obtain the asymptotic expansion of $p_{n}$ in the limit of asymptotically large $n$. The expansion was shown to have implications for the asymptotic cell shape, as well as for the finite $n$ behavior [20] and for correlations between neighboring cells [21].

A natural question to ask, then, is whether a similar asymptotic analysis of $p_{n}$ (for the Crofton cell) and of $p_{n}^{\text {typ }}$ (for the typical cell) can be carried out for the Poisson line tessellation. Now it was observed by Hug and Schneider [22] that the Crofton cell and the typical Poisson-Voronoi cell are particular instances of a more general family of zero-cell problems, dependent on a parameter $\alpha$. Hence our interest in the Poisson line tessellation leads us quite naturally to study here this full one-parameter family, which we will define in the next subsection.

\subsection{A family of tessellations}

Let $a$ be a line in the plane and $\mathbf{R}_{a}$ the projection of the origin onto that line, as shown in figure 1, then $a$ is uniquely specified by $\mathbf{R}_{a}$. In the Poisson line tessellation the $\mathbf{R}_{a}$ are independent identically distributed stochastic vectors with a density proportional to $1 / R_{a}$. This tessellation is statistically invariant under translations. In fact, the intersections of the lines $a$ of the tessellation with an arbitrary additional line constitute a one-dimensional Poisson process; and the associated angles of intersection $\theta_{a}$ are mutually independent and have the common probability density $\frac{1}{2} \sin \theta$, where $0<$ $\theta<\pi$. In this work we will consider a more general tessellation that was introduced by Hug and Schneider [22] and depends on a parameter $\alpha$. The projection vectors of this tessellation are distributed with a density

$$
\rho(\mathbf{R})=\operatorname{cst} \times R^{\alpha-2}, \quad \alpha \geq 1
$$

For generic $\alpha$ the distribution (1.1) has central symmetry around the origin but the corresponding tessellation is not translationally invariant. The zerocell is therefore unlike any other cell. For $\alpha=1$ we recover the Poisson line tessellation. For $\alpha=2$ the zero-cell corresponding to (1.1) is identical to the typical cell of the Poisson-Voronoi tessellation, which is easily seen as follows. For $\alpha=2$ we have $\rho(\mathbf{R})=$ cst, so that the projections themselves have a uniform density. Then, for a given configuration of projections $\left\{\mathbf{R}_{a}\right\}$, we may imagine point particles located at the set of positions $\left\{2 \mathbf{R}_{a}\right\}$, as well as an extra point particle placed in the origin. These particles constitute a Poisson point process in the plane. The Voronoi cell of the particle in the 
origin is then equal in distribution to the typical Poisson-Voronoi cell [23], while also being identical to the zero-cell of the $\alpha=2$ line tessellation.

Although not at present of any known application in physics, it is instructive to study the entire $\alpha$ dependent family of problems in order to see how it links together the two cases of greatest renown, the Crofton cell for $\alpha=1$ and the typical Poisson-Voronoi cell for $\alpha=2$.

\subsection{Results}

For the $\alpha$ dependent tessellation defined by (1.1) we construct the large- $n$ expansion of the probability $p_{n}(\alpha)$ that the zero-cell is $n$-sided. In section 2 we consider the parameter range $\alpha>1$, for which our work is a rather straightforward extension of earlier work [18] on the Voronoi cell, $\alpha=2$. The final result for $p_{n}(\alpha)$ is best expressed with the aid of the auxiliary quantity $p_{n}^{(0)}(\alpha)$ given by

$$
p_{n}^{(0)}(\alpha)=\frac{2\left(4 \pi^{2} \alpha\right)^{n-1}}{(2 n) !}, \quad \alpha \geq 1 .
$$

In section 2 we derive that when $n \rightarrow \infty$,

$$
p_{n}(\alpha) \simeq C(\alpha) p_{n}^{(0)}(\alpha), \quad \alpha>1,
$$

where the symbol $\simeq$ is defined by the classical equivalence $\left(u_{n} \simeq v_{n}\right.$ if and only if $u_{n} / v_{n} \rightarrow 1$ for $\left.n \rightarrow \infty\right)$, and where the prefactor $C(\alpha)$ is given by

$$
C(\alpha)=\prod_{q=1}^{\infty}\left(1-\frac{3-\alpha}{q^{2}}+\frac{\alpha^{2}}{q^{4}}\right)^{-1}, \quad \alpha>1
$$

Equivalently, we have that when $n \rightarrow \infty$,

$$
\log p_{n}(\alpha)=-2 n \log n+n \log \left(\pi^{2} \mathrm{e}^{2} \alpha\right)-\frac{1}{2} \log n+\log \left(\frac{C(\alpha)}{4 \pi^{5 / 2} \alpha}\right)+o(1) .
$$

For $\alpha=2$ this result reduces to the known expressions [17, 18] of the Voronoi problem. The study for $\alpha>1$ shows, however, that the Crofton cell corresponds to a singular point at the lower limit, $\alpha=1$, of the parameter range studied here. We have $\lim _{\alpha \rightarrow 1} C(\alpha)=\infty$, the divergence being due to the factor of index $q=1$ in (1.4). This signals the breakdown of the method of section 2 when applied to the Crofton cell, $\alpha=1$.

In section 3 we develop the modified approach necessary to find the sidedness probability $p_{n}(1)$ of the Crofton cell, as well as its other statistical properties. The appropriate result for the Crofton cell derived there is that for $n \rightarrow \infty$

$$
p_{n}(1) \simeq \frac{2}{3} n p_{n}^{(0)}(1)
$$


or equivalently

$$
\log p_{n}(1)=-2 n \log n+n \log \left(\pi^{2} \mathrm{e}^{2}\right)+\frac{1}{2} \log n-\log \left(6 \pi^{5 / 2}\right)+o(1) .
$$

As a corollary we find in section 4 that the typical cell in a Poisson line tessellation has a sidedness probability $p_{n}^{\text {typ }}(1)$ given by

$$
p_{n}^{\text {typ }}(1) \simeq \frac{8}{3} n^{-1} p_{n}^{(0)}(1), \quad n \rightarrow \infty,
$$

The ratio $p_{n}(1) / p_{n}^{\text {typ }}(1) \simeq \frac{1}{4} n^{2}$ is that of the area of the $n$-sided Crofton cell to the area of the average cell in a Poisson line tessellation.

The derivation of these asymptotic series for the $p_{n}(\alpha)$ is based on a perturbation expansion around the regular $n$-sided polygon centered at the origin. The prefactor $C(\alpha)$ represents the partition function of the elastic deformations of this $n$-gon, the 'elasticity' being of purely entropic origin. The deformations with $q=1$ are, at least to linear order, translations of the cell with respect to the origin. As is briefly discussed in section 5.1, the expansion actually leads to the full probability density functional, valid in the limit $n \rightarrow \infty$, of the zero-cell perimeter.

The possibility of constructing an asymptotic series by expanding around the regular $n$-gon implies that when $n \rightarrow \infty$, the shape of the $n$-sided cell tends with probability one to a circle (which we show to be of radius $R_{\mathrm{c}}=$ $\left.(n \alpha / 2 \pi)^{1 / \alpha}\right)$. Miles [24] was the first to state this property for the Crofton cell and to validate it by heuristic methods.

The approach to circularity in the limit of large sidedness $n$ as studied here resembles, but is nevertheless distinct from, many mathematical results derived in the limit of large cell size, with the size being defined in various ways. These two limits correspond to ensembles that are fully disjoint.

The conjecture originally formulated by Kendall in the early forties says that the Crofton cell becomes circular when its area goes to infinity. Calka and Schreiber 25] proved the approach to a circle when the radius of the largest possible inscribed disk tends to infinity. Hug, Reitzner and Schneider [10] showed a generalized version of Kendall's conjecture in any dimension $d$ when the $k$-dimensional volume of the cell, in the sense of Hausdorff ( $2 \leq k \leq$ $d)$, goes to infinity. Hug et Schneider [22] considered the zero-cell of a general class of $d$-dimensional tessellations which includes the two-dimensional family studied in this work, but also includes cells ('polytopes') resulting from more general direction dependent Poisson hyperplane processes. These authors prove the approach to a limit shape when the size of the cell, as measured in any of a variety of ways, tends to infinity.

The present work, in summary, considers a special subclass of the systems introduced in reference [22] and distinguishes itself from that work in that it takes a different large-cell limit and leads to an explicit expansion for the sidedness probability. Our expansion in negative powers of $n$ is not 
mathematically rigorous; but it is of a kind that in statistical mechanics commonly leads to exact results.

\section{A one-parameter family of line tessellations}

We consider a set of lines defined by projections $\mathbf{R}$ that are distributed in the plane $\mathbb{R}^{2}$ with density

$$
\rho(\mathbf{R})=\lambda R^{\alpha-2}, \quad R>0, \quad \alpha \geq 1,
$$

where $\lambda$ has the dimension of an inverse length to the power $\alpha$ and will be kept only as a check on the dimensionalities of our formulas. The expected number $\mathcal{N}(\alpha, L)$ of projections in a disk of radius $L$ centered at the origin is equal to

$$
\mathcal{N}(\alpha, L)=\int_{R<L} \mathrm{~d} \mathbf{R} \rho(\mathbf{R})=\frac{2 \pi \lambda L^{\alpha}}{\alpha} .
$$

In order to have a well-defined problem in the infinite plane we first consider this disk occupied by $N$ projections distributed independently according to the probability density

$$
P(\mathbf{R})=\frac{\alpha}{2 \pi L^{2}}\left(\frac{L}{R}\right)^{2-\alpha}, \quad 0<R<L .
$$

At some suitable point below we will take the limit $L, N \rightarrow \infty$ with $N=$ $\mathcal{N}(\alpha, L)$.

\subsection{Sidedness probability $p_{n}(\alpha)$ of the zero-cell}

Each projection $\mathbf{R}_{a}$, for $a=1,2, \ldots, N$, has a line $a$ associated with it. Then

$p_{n}$ is the probability that $n$ lines from among the $N$ contribute a segment to the perimeter of the zero-cell (that is, the cell enclosing the origin), and that the other $N-n$ lines do not contribute segments. We will labeling these two groups of lines by $a=1,2, \ldots, n$ and $a=n+1, n+2, \ldots, N$, respectively, and abbreviate $\mathbf{R}^{[n]}=\left\{\mathbf{R}_{1}, \ldots, \mathbf{R}_{n}\right\}$. Omitting explicit indication of the dependence of $p_{n}(\alpha)$ on $N$ and $L$, we can write

$$
\begin{aligned}
p_{n}(\alpha)= & \left(\begin{array}{c}
N \\
n
\end{array}\right) \int_{R_{1}<L} \mathrm{~d} \mathbf{R}_{1} P\left(\mathbf{R}_{1}\right) \ldots \int_{R_{n}<L} \mathrm{~d} \mathbf{R}_{n} P\left(\mathbf{R}_{n}\right) \chi\left(\mathbf{R}^{[n]}\right) \\
& \times \exp \left(-\mathcal{A}_{\alpha}\left(\mathbf{R}^{[n]}\right)\right)
\end{aligned}
$$

where $\chi$ selects the $\mathbf{R}^{[n]}$ that define Crofton cells:

$$
\chi\left(\mathbf{R}^{[n]}\right)= \begin{cases}1 & \text { if } \mathbf{R}^{[n]} \text { represents an } n \text {-sided cell enclosing the origin, } \\ 0 & \text { in all other cases; }\end{cases}
$$




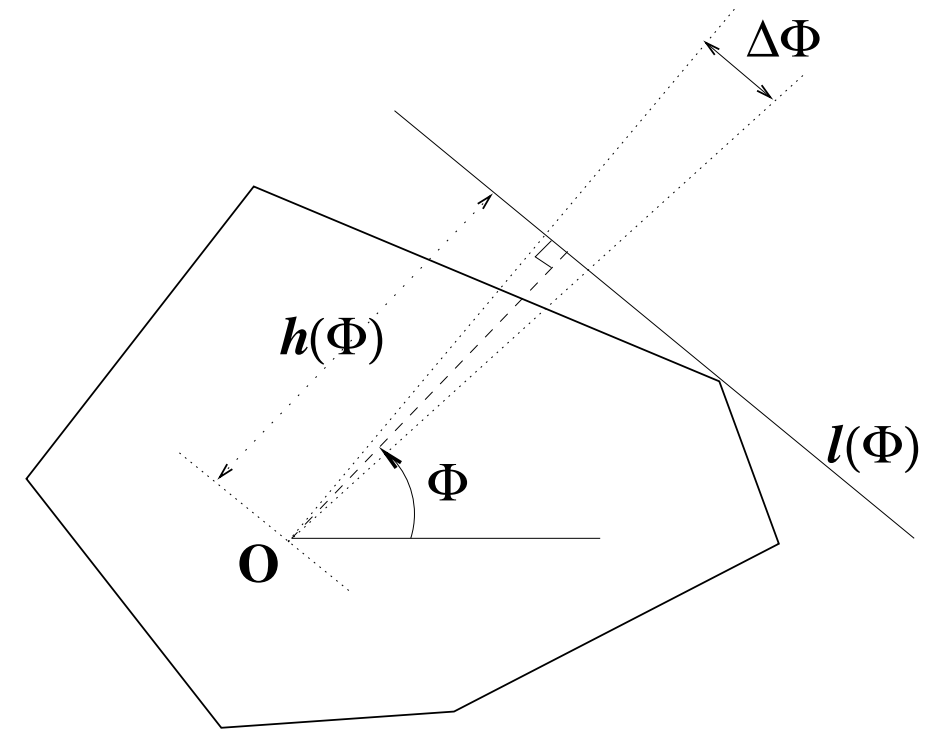

Figure 2: Figure illustrating the definition of the support function $h(\Phi)$ given in section 2.1.1.

and $\exp \left(-\mathcal{A}_{\alpha}\left(\mathbf{R}^{[n]}\right)\right)$ is the probability that the other $N-n$ lines do not intersect the perimeter of this cell. We will employ polar coordinates and write $\mathbf{R}_{a}=\left(R_{a}, \Phi_{a}\right)$. Upon using (2.3) for $P$ and partially taking the limit $N \rightarrow \infty$ we can rewrite (2.4) as

$$
p_{n}(\alpha)=\frac{\lambda^{n}}{n !} \int_{0}^{2 \pi} \mathrm{d} \Phi_{1} \ldots \mathrm{d} \Phi_{n} \int_{0}^{\infty} \mathrm{d} R_{1} R_{1}^{\alpha-1} \ldots \mathrm{d} R_{n} R_{n}^{\alpha-1} \chi\left(\mathbf{R}^{[n]}\right) \mathrm{e}^{-\mathcal{A}_{\alpha}\left(\mathbf{R}^{[n]}\right)}
$$

In order to make progress we must now render $\mathcal{A}_{\alpha}$ and $\chi$ explicit.

\subsubsection{Expression for $\mathcal{A}_{\alpha}$}

Since the $N-n$ lines are independent, $\exp \left(-\mathcal{A}_{\alpha}\right)$ is an $(N-n)$ th power and we need to consider only a single line. We refer now to figure 2, For a half-line originating in the origin $\mathbf{O}$ and having an arbitrary angular direction $\Phi$, let $\ell(\Phi)$ be the tangent to the cell that intersects this half-line perpendicularly. Generically this tangent will have only a single vertex in common with the perimeter. The distance $h(\Phi)$ from the origin to $\ell(\Phi)$ is called the 'support function' 26] of $\Phi$. The no-intersection condition can now be restated as the condition that there be no projection $\mathbf{R}_{a}(a=n+1, \ldots, N)$ in the sector of width $\Delta \Phi$ within a distance $h(\Phi)$ of the origin. The probability $w(\Phi) \Delta \Phi$ for 
a given $\mathbf{R}_{a}$ to be in this sector is

$$
\begin{aligned}
w(\Phi) \Delta \Phi & =\int_{\Phi}^{\Phi+\Delta \Phi} \mathrm{d} \Phi^{\prime} \int_{0}^{h\left(\Phi^{\prime}\right)} \mathrm{d} R R P(\mathbf{R}) \\
& =\frac{\Delta \Phi}{2 \pi}\left(\frac{h(\Phi)}{L}\right)^{\alpha}+\mathcal{O}\left(\Delta \Phi^{2}\right),
\end{aligned}
$$

where we used (2.3) . The probability $\exp \left(-\mathcal{A}_{\alpha}\right)$ for the line through $\mathbf{R}_{a}$ not to intersect the cell is equal to one minus the integral on all $\Phi$ of expression (2.7). Upon raising this result to the $(N-n)$ th power and taking the limit $N \rightarrow \infty$ we obtain the desired weight $\exp \left(-\mathcal{A}_{\alpha}\right)$ with $\mathcal{A}_{\alpha}$ given by

$$
\mathcal{A}_{\alpha}\left(\mathbf{R}^{[n]}\right)=\frac{\lambda}{\alpha} \int_{0}^{2 \pi} \mathrm{d} \Phi h^{\alpha}(\Phi) .
$$

Equations (2.6) and (2.8) can also be obtained from Slivnyak's formula by the method of reference [16]. Formula (2.6) was originally included in [27. Whenever (2.8) appears below, we will set $\lambda=1$, which entails no loss of generality.

It is easy to find the explicit expression for the support function $h(\Phi)$, which follows directly from its above definition. By a permutation of the labels of the polar angles and a rotation of the coordinate system one can always arrange things such that $0=\Phi_{0}<\Phi_{1}<\ldots<\Phi_{n-1}<2 \pi$, where by convention $\Phi_{n} \equiv \Phi_{0}$. We define the $\mathbf{S}_{m}=\left(S_{m}, \Psi_{m}\right)$ as the consecutive vertices of the cell in polar coordinate representation; in particular, $\mathbf{S}_{m}$ is the intersection of the $(m-1)$-th with the $m$-th line (see figure 3 ). After that a little algebra yields the two alternative expressions

$$
\begin{aligned}
h(\Phi) & =\left[\sin \left(\Phi_{m}-\Phi_{m-1}\right)\right]^{-1}\left[R_{m-1} \sin \left(\Phi_{m}-\Phi\right)+R_{m} \sin \left(\Phi-\Phi_{m-1}\right)\right] \\
& =S_{m} \cos \left(\Psi_{m}-\Phi\right), \quad \Phi_{m-1}<\Phi<\Phi_{m}, \quad m=1, \ldots, n
\end{aligned}
$$

When one substitutes (2.9) in (2.8), a $\Phi$ integral appears which can be carried out in closed form only for integer $\alpha$. However, the large- $n$ expansion to be introduced in the course of our development will apply for arbitrary $\alpha$.

\subsubsection{Angular variables}

Other angles essential to our study may be defined in terms of the $\Phi_{m}$ and $\Psi_{m}$ and are shown in figure 3. First of all,

$$
\begin{aligned}
& \xi_{m}=\Phi_{m}-\Phi_{m-1}, \\
& \eta_{m}=\Psi_{m+1}-\Psi_{m}, \quad m=1,2, \ldots, n,
\end{aligned}
$$

with the conventions $\Phi_{n}=\Phi_{0}+2 \pi$ and $\Psi_{n}=\Psi_{0}+2 \pi$. The $\xi_{m}$ are the angles

between two consecutive projection vectors and the $\eta_{m}$ those between two 
consecutive vertex vectors; $n$-periodicity in their index $m$ will be understood. For fixed sets of angles $\xi=\left\{\xi_{m}\right\}$ and $\eta=\left\{\eta_{m}\right\}$ one may still jointly rotate the vertex vectors $\mathbf{S}_{m}$ with respect to the projection vectors $\mathbf{R}_{m}$, as this modifies only the relative angles $\beta_{m}$ and $\gamma_{m}$ (see figure 3) between the two sets. We may select any one of these relative angles and call it 'the' angle of rotation, since it will determine all others. We will select $\beta_{1}$ for this purpose; when it is given, the remaining $\beta_{m}$ and $\gamma_{m}$ can be expressed as

$$
\begin{aligned}
& \beta_{m}=\beta_{1}-\sum_{\ell=1}^{m-1}\left(\xi_{\ell}-\eta_{\ell}\right), \quad m=2, \ldots, n, \\
& \gamma_{m}=-\beta_{1}+\sum_{\ell=1}^{m-1}\left(\xi_{\ell}-\eta_{\ell}\right)+\xi_{m}, \quad m=1, \ldots, n,
\end{aligned}
$$

We now show that $\beta_{1}$ cannot be arbitrary but is in fact determined itself by the two sets $\xi$ and $\eta$. As is clear from figure [3, one can relate $R_{m}$ to $R_{m-1}$ by $R_{m}=\left(\cos \gamma_{m} / \cos \beta_{m}\right) R_{m-1}$. Upon iterating $n$ times starting from any of the $R_{m}$, periodicity imposes that we recover the initial value. Let us define $G$ by

$$
\mathrm{e}^{2 \pi G\left(\xi, \eta ; \beta_{1}\right)}=\prod_{m=1}^{n} \frac{\cos \gamma_{m}}{\cos \beta_{m}},
$$

where the notation expresses that the $2 n-1$ variables $\gamma_{1}, \beta_{2}, \gamma_{3}, \ldots, \beta_{n}$ on the right hand side should be viewed as the functions (2.11) of the $\xi_{m}$ and $\eta_{m}$ and of the 'angle of rotation' $\beta_{1}$. Because of periodicity $\beta_{1}$ must then have the special value $\beta_{1}=\beta_{*}(\xi, \eta)$ that is the solution of

$$
G\left(\xi, \eta ; \beta_{*}\right)=0 .
$$

It was shown in reference [20] that the solution of (2.13) is unique.

Finally we remark that whereas the $\xi_{m}$ and $\eta_{m}$ must be positive, the $\beta_{m}$ and $\gamma_{m}$ may have either sign.

In terms of the angles defined above, equations (2.8)-(2.9) can be made fully explicit in those cases where it is possible to carry out the integral on $\Phi$. One such case occurs for $\alpha=1$. By Cauchy's integral formula we have $\mathcal{A}_{1}=\mathcal{P}$, where $\mathcal{P}$ is the cell perimeter and has the two alternative expressions

$$
\begin{aligned}
\mathcal{P} & =\sum_{m=1}^{n} R_{m}\left[\tan \frac{1}{2} \xi_{m}+\tan \frac{1}{2} \xi_{m+1}\right] \\
& =\sum_{m=1}^{n} R_{m}\left[\tan \gamma_{m}+\tan \beta_{m+1}\right]
\end{aligned}
$$

For $\alpha=2$ we have that $\mathcal{A}_{2}$ is the area of the fundamental domain of the Voronoi cell, that is, of the union of $n$ disks of radii $S_{m}$ centered at the vertices $\mathbf{S}_{m}$. Explicit expressions for $\mathcal{A}_{2}$ may be found in references [16, 28, 17, 18, 20]. 


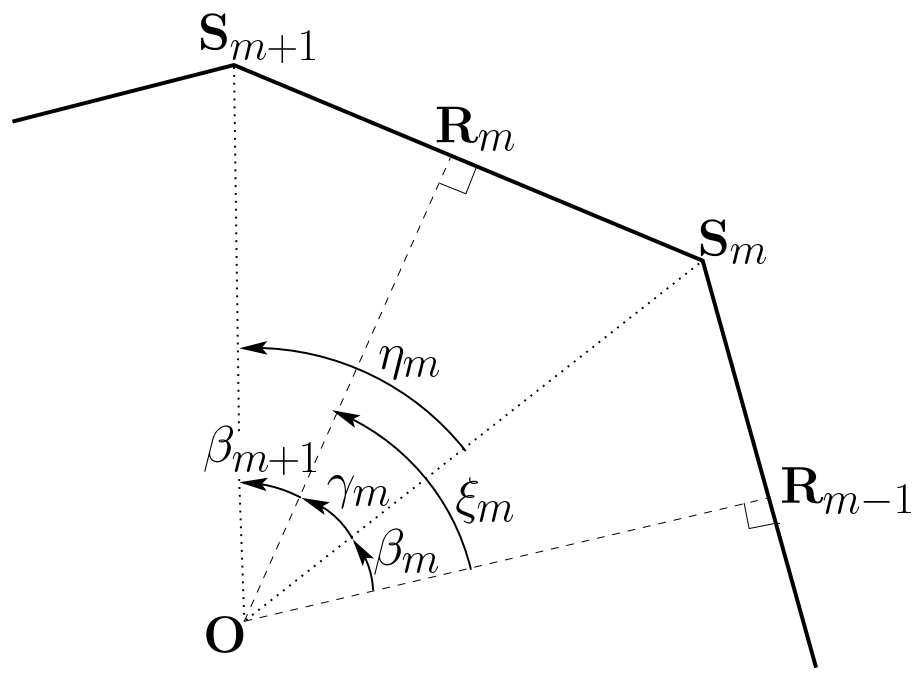

Figure 3: Heavy line segments: the perimeter of the zero-cell in a random line tessellation of the plane. The $\mathbf{R}_{m}$ are the projections of the origin onto the lines containing these perimeter segments. The $\mathbf{S}_{m}$ are the cell vertices. The figure shows the angles $\xi_{m}, \eta_{m}, \beta_{m}$, and $\gamma_{m}$ defined by (2.10) and (2.11).

\subsubsection{Expression for the indicator function $\chi$}

Having defined the angular variables we return now to the conditions imposed on the domain of integration in (2.6) by the indicator $\chi$. In terms of the angles $\beta_{m}$ and $\gamma_{m}$ these conditions simplify greatly and take the explicit form [17, 18]

$$
-\frac{\pi}{2}<\beta_{m}, \gamma_{m}<\frac{\pi}{2}, \quad \beta_{m}+\gamma_{m}>0, \quad \gamma_{m}+\beta_{m+1}>0,
$$

where $m=1,2, \ldots, n$ and $n$-periodicity in $m$ is understood. As could be expected, this condition depends only on the angles of the problem and not on the length scale.

\subsection{Transforming the expression for $p_{n}(\alpha)$}

Equation (2.6) represents $p_{n}(\alpha)$ as a $2 n$-fold integral which we will transform in successive steps to a form manageable in the large $n$ limit. An important variable is the 'average radius' $R_{\mathrm{av}}$ defined by

$$
R_{\mathrm{av}}=\frac{1}{n} \sum_{m=1}^{n} R_{m} .
$$

We now pass from the set of variables of integration $\left\{R_{m}, \Phi_{m}\right\}$ used in (2.6) to a new set of variables consisting of the radial scale $R_{\mathrm{av}}$ and the set of angles $\left\{\xi_{m}, \eta_{m}\right\}$. Although the $\beta_{m}$ and $\gamma_{m}$ may be entirely expressed in terms of the $\xi_{\ell}$ and $\eta_{\ell}$, they constitute a useful set of auxiliary variables. 
Convenient shorthand notation will be

$$
\int_{\xi, \eta} \equiv \int_{0}^{2 \pi} \mathrm{d} \xi_{1} \xi_{1} \ldots \mathrm{d} \xi_{n} \xi_{n} \int_{0}^{2 \pi} \mathrm{d} \eta_{1} \ldots \mathrm{d} \eta_{n} \delta\left(\sum_{m=1}^{n} \xi_{m}-2 \pi\right) \delta\left(\sum_{m=1}^{n} \eta_{m}-2 \pi\right)
$$

and

$$
\Theta \equiv \prod_{m=1}^{n} \theta\left(\frac{\pi}{2}-\beta_{m}\right) \prod_{m=1}^{n} \theta\left(\frac{\pi}{2}-\gamma_{m}\right)
$$

where $\theta$ is the Heaviside unit step function. After doing the algebra of the transformation of variables [18, 20] we find that (2.6) may be recast in the form

$$
p_{n}(\alpha)=\frac{1}{n} \int_{\xi, \eta} G^{\prime}\left(\xi, \eta ; \beta_{*}\right)^{-1}\left[\prod_{m=1}^{n} \rho_{m}^{\alpha} T_{m} \xi_{m}^{-1}\right] \Theta \int_{0}^{\infty} \mathrm{d} R_{\mathrm{av}} R_{\mathrm{av}}^{\alpha n-1} \mathrm{e}^{-\mathcal{A}_{\alpha}},
$$

in which $G^{\prime}$ denotes the derivative of $G\left(\xi, \eta ; \beta_{1}\right)$ with respect to $\beta_{1}$; we have abbreviated $T_{m} \equiv \sin \xi_{m} / \cos ^{2} \beta_{m}$; and the $2 n$ ratios $\rho_{m} \equiv R_{m} / R_{\text {av }}$ may be expressed in terms of the angles by means of the $2 n$ relations

$$
\rho_{m}=\left(\cos \gamma_{m} / \cos \beta_{m}\right) \rho_{m-1} \quad(m=2, \ldots, n), \quad n^{-1} \sum_{m=1}^{n} \rho_{m}=1,
$$

of which the last one follows from (2.16).

Clearly $\mathcal{A}_{\alpha}$ can be written as $R_{\mathrm{av}}^{\alpha}$ times a function of the angles. We will set

$$
\mathcal{A}_{\alpha}=\frac{2 \pi}{\alpha} R_{\mathrm{av}}^{\alpha}\left(1+n^{-1} V\right),
$$

where $2 \pi \alpha^{-1} R_{\mathrm{av}}^{\alpha}$ is the value that $\mathcal{A}_{\alpha}$ takes for a circular cell of radius $R_{\mathrm{av}}$ and in which $V$ depends exclusively on the angles. The notation in (2.21) is meant to suggest that $n^{-1} V$ is of order $n^{-1}$ as $n \rightarrow \infty$, but we will rely on that only later. Integrating (2.19) on $R_{\mathrm{av}}$ converts it into

$$
p_{n}(\alpha)=\frac{(n-1) !}{2 \pi n}\left(\frac{\alpha}{2 \pi}\right)^{n-1} \int_{\xi, \eta} \Theta \mathrm{e}^{-\mathbb{V}(\alpha)}, \quad \alpha \geq 1
$$

where

$$
\mathrm{e}^{-\mathbb{V}(\alpha)}=G^{\prime}\left(\xi, \eta ; \beta_{*}\right)^{-1}\left[\prod_{m=1}^{n} \rho_{m}^{\alpha} T_{m} \xi_{m}^{-1}\right]\left(1+n^{-1} V\right)^{-n}
$$

The integrations that remain on the right hand side of (2.22) bear only on the angles $\xi_{m}$ and $\eta_{m}$, that is, on the shape of the cell without regard to its radial dimension. We still remark that the factors $\xi_{m}^{-1}$ in the product on $m$ in (2.23) compensate the factors $\xi_{m}$ incorporated in the definition (2.17) of $\int_{\xi, \eta}$. Their purpose is to ensure that $\mathbb{V}(\alpha)$ remains finite when any of the $\xi_{m}$ tends to zero. 
We finally write $(2.22)$ as

$$
p_{n}(\alpha)=p_{n}^{(0)}(\alpha)\left\langle\Theta \mathrm{e}^{-\mathbb{V}(\alpha)}\right\rangle, \quad \alpha \geq 1,
$$

where for any function $X$ of the angular variables we define

$$
\langle X\rangle=\frac{\int_{\xi, \eta} X}{\int_{\xi, \eta} 1} .
$$

Straightforward calculation yields

$$
\begin{aligned}
p_{n}^{(0)}(\alpha) & =\frac{(n-1) !}{2 \pi n}\left(\frac{\alpha}{2 \pi}\right)^{n-1} \int_{\xi, \eta} 1 \\
& =2 \frac{\left(4 \pi^{2} \alpha\right)^{n-1}}{(2 n) !}, \quad \alpha \geq 1
\end{aligned}
$$

which is equation (1.2) of the introduction. Equations (2.24) and (2.26) represent an important step forward with respect to the initial expressions (2.6) and (2.8). However, the hard part of the problem remains, namely

to determine the $n$ dependence of $\left\langle\Theta \mathrm{e}^{-\mathbb{V}(\alpha)}\right\rangle$ in equation (2.24). In sections 2.42 .5 we will show that for $\alpha>1 \lim _{n \rightarrow \infty}\left\langle\Theta \mathrm{e}^{-\mathbb{V}(\alpha)}\right\rangle=C(\alpha)$, where $C(\alpha)$ is a finite numerical constant. The special case of the Crofton cell, $\alpha=1$, will not be covered by the arguments of those sections and must be dealt with separately; we will do so in section 3 .

\subsection{Scaling of the average radius $R_{\mathrm{av}}$}

The inner integrand of (2.19), when combined with (2.21), shows that the average radius $R_{\mathrm{av}}$ has a probability distribution

$$
P_{\mathrm{av}}\left(R_{\mathrm{av}}\right)=\operatorname{cst} \times R_{\mathrm{av}}^{\alpha n-1} \mathrm{e}^{-(2 \pi / \alpha) R_{\mathrm{av}}^{\alpha}\left(1+n^{-1} V\right)}, \quad \alpha \geq 1,
$$

which depends on the cell shape through $V$. The notation is meant to suggest that $n^{-1} V$ is negligible when $n \rightarrow \infty$, and this will be confirmed below. Elementary analysis then shows that for $n \rightarrow \infty$ the distribution $P_{\mathrm{av}}\left(R_{\mathrm{av}}\right)$ has a peak at $R_{\mathrm{c}}$ whose width is $\sigma_{\mathrm{c}}$, where

$$
R_{\mathrm{c}}=\left(\frac{n \alpha}{2 \pi}\right)^{\frac{1}{\alpha}}, \quad \sigma_{\mathrm{c}}=\frac{1}{\alpha}\left(\frac{\alpha}{2 \pi}\right)^{\frac{1}{\alpha}} n^{\frac{1}{\alpha}-\frac{1}{2}} .
$$

Hence the typical deviation of $R_{\mathrm{av}}$ from $R_{\mathrm{c}}$ is a factor $n^{\frac{1}{2}}$ smaller than $R_{\mathrm{c}}$ itself, for all $\alpha \geq 1$. 


\subsection{Large- $n$ expansion of $\mathbb{V}(\alpha)$}

The regular $n$-sided polygon is a point of high symmetry in phase space where we expect the integrand of $\int_{\xi, \eta}$ in (2.22) to be stationary. This point has $\xi_{m}=\eta_{m}=2 \pi n^{-1}$ and $\beta_{m}=\gamma_{m}=\pi n^{-1}$ for all $m=1,2, \ldots, n$. Coordinates that describe the deviations from this symmetric state are defined by

$$
\delta \xi_{m}=\xi_{m}-2 \pi n^{-1}, \quad \delta \eta_{m}=\eta_{m}-2 \pi n^{-1}
$$

and their suitably scaled Fourier transforms

$$
\hat{X}_{q}=\frac{n^{\frac{1}{2}}}{2 \pi} \sum_{m=1}^{n} \mathrm{e}^{2 \pi \mathrm{i} q m / n} \delta \xi_{m}, \quad \hat{Y}_{q}=\frac{n^{\frac{1}{2}}}{2 \pi} \sum_{m=1}^{n} \mathrm{e}^{2 \pi \mathrm{i} q m / n} \delta \eta_{m},
$$

where $q= \pm 1, \pm 2, \ldots$. It will appear that the integrations required for the calculation of the average in (2.24) can be done explicitly once expressed in terms of the variables of integration $\hat{X}_{q}$ and $\hat{Y}_{q}$.

\subsubsection{Scaling with $n$}

We will treat $\mathbb{V}$ perturbatively in inverse powers of $n$. An initial hypothesis on the smallness of the angles with $n$ is suggested by the scaling that prevails when in (2.22) we set $\mathbb{V}=0$. That yields

$$
\delta \xi_{m}, \delta \eta_{m} \sim n^{-1}
$$

where the symbol $\sim$ indicates the scaling with $n$ in the large- $n$ limit. Using relations (2.11) and (2.20) between the angles as well as the fact that the $\xi_{m}$ and $\eta_{m}$ are to leading order independent, we find from (2.31) that furthermore

$$
\beta_{m}, \gamma_{m} \sim n^{-\frac{1}{2}}, \quad \tau_{m} \equiv \rho_{m}-1 \sim n^{-\frac{1}{2}} ;
$$

and using (2.30) we find from (2.29) that

$$
\hat{X}_{q} \sim n^{0}, \quad \hat{Y}_{q} \sim n^{0} .
$$

If and when needed, the $\beta_{m}, \gamma_{m}$, and $\tau_{m}$ and their Fourier transforms can be expressed in terms of the $\hat{X}_{q}$ and $\hat{Y}_{q}$.

The initial hypothesis now holds that the above scalings remain valid in the presence of the nonzero $\mathbb{V}$ defined by (2.23). We will consider the validity of this hypothesis confirmed when at the end of this section we will find that in the limit $n \rightarrow \infty$ it produces a finite leading order result for $\langle\exp (-\mathbb{V})\rangle$.

We end these considerations with two remarks. First, the present scalings are identical to those encountered in the study of the large $n$-sided Voronoi cell. How to handle them technically, in particular when sums of $\sim n$ terms appear, is nontrivial and has been discussed in detail in reference [18], sections 5 and 6 . Secondly, we note that the smallness of the $\tau_{m}$ with $n$ implies that all $R_{m}$ are close to the average $R_{\mathrm{av}}$, which in turn together with the smallness of the $\xi_{m}$ implies that for $n \rightarrow \infty$ the perimeter approaches a circle. 


\subsubsection{The expansion}

We defined $\mathbb{V}$ recursively by (2.23), (2.21), and (2.8) in terms of $V, \mathcal{A}_{\alpha}$, and $h(\Phi)$. Since $h(\Phi)$ defined by (2.9) is piecewise analytic on $n$ successive angular intervals, when substituted in (2.8) it gives rise to a sum of $n$ terms. In the $m$ th term, that is, for $\Phi_{m-1}<\Phi<\Phi_{m}$, we set $S_{m}=R_{m} / \cos \gamma_{m}$ and pass to the variable of integration $\theta \equiv \Phi-\Psi_{m}$. Then (2.8) becomes

$$
\begin{aligned}
\mathcal{A}_{\alpha} & =\frac{1}{\alpha} \sum_{m=1}^{n}\left(\frac{R_{m}}{\cos \gamma_{m}}\right)^{\alpha} \int_{-\beta_{m}}^{\gamma_{m}} \mathrm{~d} \theta(\cos \theta)^{\alpha} \\
& =\frac{R_{\mathrm{av}}^{\alpha}}{\alpha} \sum_{m=1}^{n}\left(\frac{1+\tau_{m}}{1-\frac{1}{2} \gamma_{m}^{2}+\ldots}\right)^{\alpha} \int_{-\beta_{m}}^{\gamma_{m}} \mathrm{~d} \theta\left(1-\frac{1}{2} \theta^{2}+\ldots\right)^{\alpha} .
\end{aligned}
$$

Here the dots indicate terms of higher order in the angles. We may expand the $\alpha$ th powers in (2.34) and do the $\theta$ integral. Using the sum rule $\sum_{m=1}^{n}\left(\beta_{m}+\right.$ $\left.\gamma_{m}\right)=2 \pi$ we find that the leading order result for $\mathcal{A}_{\alpha}$ is $(2 \pi / \alpha) R_{\mathrm{av}}^{\alpha}$. Comparison to (2.21) shows that the higher order terms in (2.34) determine $V$. Pursuing the expansion to higher orders we find

$$
\begin{aligned}
\frac{2 \pi}{n} V= & \alpha \sum_{m=1}^{n} \tau_{m}\left(\gamma_{m}+\beta_{m}\right)+\frac{1}{2} \alpha(\alpha-1) \sum_{m=1}^{n} \tau_{m}^{2}\left(\gamma_{m}+\beta_{m}\right) \\
& +\frac{1}{6} \alpha\left(2 \gamma_{m}^{3}+3 \gamma_{m}^{2} \beta_{m}-\beta_{m}^{3}\right)+\ldots
\end{aligned}
$$

Equation (2.35) for $V$ may be substituted in (2.23). The other factors in (2.23) may be expanded similarly, and together this leads to the expansion of $\mathbb{V}$. The order in $n$ of each term in the expansion may be estimated in the way outlined in section 2.4.1. It then appears that $\mathbb{V}(\alpha)$ allows for an expansion in powers of $n^{-\frac{1}{2}}$,

$$
\mathbb{V}(\alpha)=\mathbb{V}_{1}(\alpha)+\mathcal{O}\left(n^{-\frac{1}{2}}\right)
$$

of which the leading term $\mathbb{V}_{1}(\alpha)$ is a quadratic form in the angles that is of order $n^{0}$. Upon expressing all variables in terms of the $\hat{X}_{q}$ and $\hat{Y}_{q}$ one obtains

$$
\mathbb{V}_{1}(\alpha)=\sum_{q \neq 0}\left(\hat{X}_{q}, \hat{Y}_{q}\right) \cdot \mathbf{V}_{q} \cdot\left(\hat{X}_{-q}, \hat{Y}_{-q}\right)^{\mathrm{T}}
$$

where the superscript $\mathrm{T}$ indicates transposition and where $\mathbf{V}_{q}$ is the symmetric matrix

$$
\mathbf{V}_{q}=\left(\begin{array}{cc}
A_{q} & -A_{q}+\frac{1}{2} B_{q} \\
-A_{q}+\frac{1}{2} B_{q} & A_{q}-B_{q}
\end{array}\right)
$$

with

$$
A_{q}=\frac{\alpha-1}{q^{2}}+\frac{\alpha^{2}}{2 q^{4}}, \quad B_{q}=\frac{\alpha}{q^{2}} .
$$

Equations (2.36)-(2.39) complete the large- $n$ expansion of $\mathbb{V}(\alpha)$. They are valid for all $\alpha \geq 1$. 


\subsection{Large- $n$ expansion of $p_{n}(\alpha)$}

The large- $n$ expansion of $p_{n}(\alpha)$ is based on the one of $\mathbb{V}$ given above. Upon substituting (2.36)-(2.37) in (2.24) and using that $\Theta$ may be replaced with unity up to corrections that vanish exponentially for large $n$, we get

$$
p_{n}(\alpha) \simeq p_{n}^{(0)}(\alpha)\left\langle\mathrm{e}^{-\mathbb{V}_{1}(\alpha)}\right\rangle
$$

with $\mathbb{V}_{1}$ given by (2.37).

Although (2.37) is a quadratic form, the $\hat{X}_{q}$ and $\hat{Y}_{q}$ are not Gaussian distributed. Nevertheless, it was shown in reference [18] that to leading order in $n^{-1 / 2}$ the average $\langle\ldots\rangle$ in (2.40) may be carried out as though the $\hat{X}_{q}$ and $\hat{Y}_{q}$ were Gaussian, with a probability distribution

$$
\mathcal{N}_{G} \exp \left(-\sum_{q \neq 0}\left(\hat{X}_{q}, \hat{Y}_{q}\right) \cdot \mathbf{E}^{-1} \cdot\left(\hat{X}_{-q}, \hat{Y}_{-q}\right)^{\mathrm{T}}\right),
$$

where $\mathcal{N}_{G}$ is the appropriate normalization constant and where we introduced the $2 \times 2$ diagonal matrix $\mathbf{E}=\operatorname{diag}\{1,2\}$. This fact is partly confirmed by the result of convergence in distribution and asymptotic mutual independence of the Fourier coefficients of i.i.d. random variables [29, 30]. The Gaussian integrals are easily done. Still letting 1 represent the $2 \times 2$ unit matrix one finds that

$$
p_{n}(\alpha) \simeq C(\alpha) p_{n}^{(0)}(\alpha), \quad C(\alpha)=\prod_{q=1}^{\infty} \Lambda_{q}^{-1}(\alpha), \quad \alpha>1,
$$

where we abbreviated for all $\alpha \geq 1$

$$
\begin{aligned}
\Lambda_{q}(\alpha) & =\operatorname{det}\left(\mathbf{1}+\mathbf{V}_{q} \mathbf{E}\right) \\
& =1+3 A_{q}-2 B_{q}-\frac{1}{2} B_{q}^{2} \\
& =1-(3-\alpha) q^{-2}+\alpha^{2} q^{-4} .
\end{aligned}
$$

Equations (2.42) and (2.43) are the main result of this section. It is easily verified that $\Lambda_{q}(\alpha)>0$ for all $\alpha \geq 1$ and $q=1,2, \ldots$ with the only exception that $\Lambda_{1}(1)=0$. This is the reason why the substitution that led to (2.40) must be restricted to $\alpha>1$; for the Crofton point $\alpha=1$ the resulting Gaussian integration would diverge. Hence the method of this section leaves the Crofton problem unsolved. In the following section we will develop a modified method suitable for the Crofton cell.

\section{The Crofton cell: $\alpha=1$}

\subsection{Centering condition}

For $\alpha=1$ the expansion of $\left\langle\Theta \mathrm{e}^{-\mathbb{V}(\alpha)}\right\rangle$ around a regular $n$-gon leads to a diverging integral and hence cannot be used for finding $p_{n}(1)$. This divergence, 
due to the vanishing of $\Lambda_{q}(1)$ with $q=1$, may be tracked down to the origin being located with the same probability in any point of the cell; hence the problem has a 'zero mode' due to translational invariance. Indeed, for $q= \pm 1$ elastic deformations of a circle are actually translations.

This analysis also points the way out of the difficulty. In the integrals one should count a Crofton cell only when, under a suitable definition, it is 'centered' around the origin, and then give it a weight proportional to its area in order to simultaneously account for all noncentered cells that may be obtained from it by translation. The 'centering condition' that we adopt is that the $q= \pm 1$ Fourier components of $R_{m}$ vanish, that is,

$$
\hat{R}_{1} \equiv \frac{1}{n} \sum_{m=1}^{n} \mathrm{e}^{2 \pi \mathrm{i} m / n} R_{m}=0 .
$$

This is a constraint only on the vector lengths $R_{m}$, irrespective of the angles $\Phi_{m}$.

The following remark establishes one more link with the mathematical literature. There, a function $\mathcal{A}_{2}\left(\mathbf{R}^{[n]} ; \mathbf{r}\right)$ appears defined as $\mathcal{A}_{\alpha}\left(\mathbf{R}^{[n]}\right)$ with $\alpha=2$ in (2.8) except that the support function $h(\Phi)$ with respect to the origin $\mathbf{O}$ is replaced by the support function $h(\Phi ; \mathbf{r})$ with respect to $\mathbf{r}$. The function $\mathcal{A}_{2}\left(\mathbf{R}^{[n]} ; \mathbf{r}\right)$ is commonly used to calculate the probability that a convex set containing the origin translated by $-\mathbf{r}$ is included in the typical Poisson-Voronoi cell. As a function of $\mathbf{r}, \mathcal{A}_{2}\left(\mathbf{R}^{[n]} ; \mathbf{r}\right)$ is a convex function such that its gradient $\nabla_{\mathbf{r}} \mathcal{A}_{2}\left(\mathbf{R}^{[n]} ; \mathbf{r}\right)$ is, to, up to a multiplicative constant, equal to $\left(\int_{0}^{2 \pi} h(\Phi ; \mathbf{r}) \cos \Phi d \Phi, \int_{0}^{2 \pi} h(\Phi ; \mathbf{r}) \sin \Phi d \Phi\right)$. The sum appearing in (3.1) is the discrete version of this gradient. Hence (3.1) amounts to adopting as the 'center' of the cell the point for which the fundamental domain has minimum area; we remark that this point is given by $\left(\pi^{-1} \int_{0}^{2 \pi} h(\Phi) \cos \Phi d \Phi, \pi^{-1} \int_{0}^{2 \pi} h(\Phi) \sin \Phi d \Phi\right)$.

In the presence of constraint (3.1) the necessary mathematics requires a few preliminaries. Let $\mathbf{t}=\left(t_{x}, t_{y}\right)$ be a translation applied to a Crofton cell. Under $\mathbf{t}$ the vertices $\mathbf{S}_{m}$ are translated by just that amount. However, the $\mathbf{R}_{m}$, being projections of the origin onto lines that under the translation stay parallel to themselves, transform differently. If $\mathbf{t}$ keeps the origin inside the cell, the $\mathbf{R}_{m}$ transform into $\mathbf{R}_{m, \mathbf{t}}=\left(R_{m, \mathbf{t}}, \Phi_{m, \mathbf{t}}\right)$ given by

$$
\begin{aligned}
R_{m, \mathbf{t}} & =R_{m}+t_{x} \cos \Phi_{m}+t_{y} \sin \Phi_{m}, \\
\Phi_{m, \mathbf{t}} & =\Phi_{m} .
\end{aligned}
$$

We will write $\mathbf{R}_{\mathbf{t}}^{[n]}=\left\{\mathbf{R}_{1, \mathbf{t}}, \ldots, \mathbf{R}_{n, \mathbf{t}}\right\}$. It will be convenient to extend the definition (3.2) of $\mathbf{R}_{\mathbf{t}}^{[n]}$ to translations $\mathbf{t}$ that take the origin out of the cell. In that case, however, $\mathbf{R}_{\mathbf{t}}^{[n]}$ is 'nonphysical' (it does not represent a Crofton cell any more; one or more of its $R_{m, \mathbf{t}}$ are negative) and, by virtue of (2.5), the indicator $\chi\left(\mathbf{R}_{\mathbf{t}}^{[n]}\right)$ then vanishes. 
We now show that if $\mathbf{R}^{[n]}$ defines a Crofton cell, then there exists a unique 'centering translation' $\mathbf{t}^{*}\left(\mathbf{R}^{[n]}\right)$, that is, one for which (3.1) is satisfied:

$$
\hat{R}_{1, \mathbf{t}^{*}}=0 \text {. }
$$

The proof goes by explicit construction: using (3.1) and (3.2) in (3.3) leads to a linear system of equations for $\mathbf{t}^{*}$ whose solution is

$$
\mathbf{t}^{*}\left(\mathbf{R}^{[n]}\right)=-\frac{1}{2} \sqrt{2} n \boldsymbol{\varphi}^{-1}\left(\begin{array}{c}
\hat{R}_{1}^{\mathrm{c}} \\
\hat{R}_{1}^{\mathrm{s}}
\end{array}\right)
$$

where for any complex variable $z$ we set $z=\left(z^{\mathrm{c}}+\mathrm{i} z^{\mathrm{s}}\right) / \sqrt{2}$ and where $\varphi$ is the matrix of elements

$$
\begin{aligned}
\varphi_{11} & =\sum_{m} \cos (2 \pi m / n) \cos \Phi_{m}, & \varphi_{12} & =\sum_{m} \cos (2 \pi m / n) \sin \Phi_{m} \\
\varphi_{21} & =\sum_{m} \sin (2 \pi m / n) \cos \Phi_{m}, & \varphi_{22} & =\sum_{m} \sin (2 \pi m / n) \sin \Phi_{m} .
\end{aligned}
$$

Two useful relations,

$$
\mathbf{t}^{*}\left(\mathbf{R}_{\mathbf{t}}^{[n]}\right)=\mathbf{t}^{*}\left(\mathbf{R}^{[n]}\right)-\mathbf{t},
$$

and

$$
\int_{\mathbb{R}^{2}} \mathrm{~d} \mathbf{t} \delta\left(\mathbf{t}-\mathbf{t}^{*}\left(\mathbf{R}^{[n]}\right)\right) \chi\left(\mathbf{R}_{\mathbf{t}}^{[n]}\right)=1
$$

will serve below.

\subsection{Modified starting point for $\alpha=1$}

After these preliminaries we return to the general expression (2.6) for the sidedness probability $p_{n}(\alpha)$. For the case of the Crofton cell we insert (3.7) and rewrite $p_{n}(1)$ as

$$
\begin{aligned}
p_{n}(1)= & \frac{\lambda^{n}}{n !} \int_{0}^{2 \pi} \mathrm{d} \Phi_{1} \ldots \mathrm{d} \Phi_{n} \int_{\mathbb{R}^{2}} \mathrm{~d} \mathbf{t} \int_{0}^{\infty} \mathrm{d} R_{1} \ldots \mathrm{d} R_{n} \\
& \times \delta\left(\mathbf{t}-\mathbf{t}^{*}\left(\mathbf{R}^{[n]}\right)\right) \chi\left(\mathbf{R}^{[n]}\right) \chi\left(\mathbf{R}_{\mathbf{t}}^{[n]}\right) \mathrm{e}^{-\mathcal{P}\left(\mathbf{R}^{[n]}\right)},
\end{aligned}
$$

where we used that $\mathcal{A}_{1}=\mathcal{P}$ and have indicated explicitly the dependence on $\mathbf{R}^{[n]}$ of all quantities involved.

Under the $\mathbf{t}$ integral in (3.8) we now transform from the $R_{m}$ to new radial variables of integration $R_{m}^{\prime} \equiv R_{m, \mathbf{t}}$, with the $R_{m, \mathbf{t}}$ given by (3.2). We may express $\mathbf{R}^{[n]}$ in terms of $\mathbf{R}^{\prime[n]}$ by means of $\mathbf{R}^{[n]}=\mathbf{R}_{-\mathbf{t}}^{\prime[n]}$. The Jacobian of this transformation is unity but the limits of integration should be treated with some care. Equation (3.2) implies for $R_{m}^{\prime}$ the domain of integration 
$t_{x} \cos \Phi_{m}+t_{y} \sin \Phi_{m}<R_{m}^{\prime}<\infty$. If the lower limit of integration is negative, we may replace it with zero because $\chi\left(\mathbf{R}_{\mathbf{t}}^{[n]}\right)=0$ on the interval discarded; and if the lower integration limit is positive, we may also replace it with zero, because then $\chi\left(\mathbf{R}^{[n]}\right)=0$ on the interval added to the domain of integration. Hence, still using (3.6), we get

$$
\begin{aligned}
p_{n}(1)= & \frac{\lambda^{n}}{n !} \int_{0}^{2 \pi} \mathrm{d} \Phi_{1} \ldots \mathrm{d} \Phi_{n} \int_{\mathbb{R}^{2}} \mathrm{~d} \mathbf{t} \int_{0}^{\infty} \mathrm{d} R_{1}^{\prime} \ldots \mathrm{d} R_{n}^{\prime} \\
& \times \delta\left(\mathbf{t}^{*}\left(\mathbf{R}^{\prime[n]}\right)\right) \chi\left(\mathbf{R}^{\prime[n]}\right) \chi\left(\mathbf{R}_{-\mathbf{t}}^{\prime[n]}\right) \mathrm{e}^{-\mathcal{P}\left(\mathbf{R}_{-\mathbf{t}}^{\prime[n]}\right)} .
\end{aligned}
$$

Since the cell perimeter is invariant under translation, we have $\mathcal{P}\left(\mathbf{R}_{-\mathbf{t}}^{\prime[n]}\right)=$ $\mathcal{P}\left(\mathbf{R}^{\prime[n]}\right)$. The only $\mathbf{t}$ dependence left in the integrand of $(3.9)$ is then the one in $\chi\left(\mathbf{R}_{-\mathbf{t}}^{\prime[n]}\right)$. The integral on $\mathbf{t}$ of this quantity has a nonzero contribution only in a domain of the same size and shape as the cell itself and therefore produces the cell area $A\left(\mathbf{R}^{\prime[n]}\right)$. Hence, still suppressing the primes, we get from (3.9)

$$
\begin{aligned}
p_{n}(1)= & \frac{\lambda^{n}}{n !} \int_{0}^{2 \pi} \mathrm{d} \Phi_{1} \ldots \mathrm{d} \Phi_{n} \int_{0}^{\infty} \mathrm{d} R_{1} \ldots \mathrm{d} R_{n} \\
& \times \delta\left(\mathbf{t}^{*}\left(\mathbf{R}^{[n]}\right)\right) \chi\left(\mathbf{R}^{[n]}\right) A\left(\mathbf{R}^{[n]}\right) \mathrm{e}^{-\mathcal{P}\left(\mathbf{R}^{[n]}\right)},
\end{aligned}
$$

Equation (3.10) constitutes a modified starting point for the calculation of the sidedness probability $p_{n}(1)$ of the Crofton cell. It differs from the original expression (2.6) by the insertion of a delta function and of the area factor $A\left(\mathbf{R}^{[n]}\right)$.

\subsection{Sidedness probability $p_{n}(1)$ of the Crofton cell}

Having rewritten the definition of the sidedness probability $p_{n}(1)$ as (3.10), we are now in a position to start its explicit evaluation. The cell area $A$ is given by

$$
A=R_{\text {av }}^{2} A_{\text {ang }}, \quad A_{\text {ang }}=\frac{1}{2} \sum_{m=1}^{n} \rho_{m}^{2}\left(\tan \gamma_{m}+\tan \beta_{m+1}\right),
$$

where $A_{\text {ang }}$ is an expression only in terms of the angular variables. We may similarly isolate the radial part of the centering translation $\mathbf{t}^{\star}$ found in (3.4) by writing

$$
\mathbf{t}^{\star}=R_{\mathrm{av}}^{2} \mathbf{t}_{\mathrm{ang}}^{\star},
$$

where $\mathbf{t}_{\text {ang }}^{\star}$ depends only on the angles.

When we substitute (3.11) and (3.12) in (3.10), the factors $R_{\text {av }}$ stemming from $\delta\left(\mathbf{t}^{\star}\right)$ and from the area $A$ cancel. We set $\mathcal{P}=2 \pi R_{\text {av }}\left(1+n^{-1} V\right)$, which 
is the special case $\alpha=1$ of (2.21); here $2 \pi R_{\mathrm{av}}$ is the perimeter of a circular cell of radius $R_{\mathrm{av}}$. We may in the same way as before pass to the variables $\left\{\xi_{m}, \eta_{m}\right\}$ and a single length scale $R_{\mathrm{av}}$. Carrying out the $R_{\mathrm{av}}$ integration we get, setting $\lambda=1$ as before,

$$
\begin{aligned}
p_{n}(1) & =\frac{1}{n !} \int_{0}^{2 \pi} \mathrm{d} \Phi_{1} \ldots \mathrm{d} \Phi_{n} \int_{0}^{\infty} \mathrm{d} R_{1} \ldots \mathrm{d} R_{n} \delta\left(\mathbf{t}_{\text {ang }}^{*}\right) A_{\text {ang }} \chi \mathrm{e}^{-\mathcal{P}} \\
& =\frac{1}{n} \int_{\xi, \eta} G^{\prime}\left(\xi, \eta ; \beta_{*}\right)^{-1}\left[\prod_{m=1}^{n} \rho_{m}^{\alpha} T_{m} \xi_{m}^{-1}\right] \Theta \int_{0}^{\infty} \mathrm{d} R_{\text {av }} R_{\text {av }}^{n-1} \mathrm{e}^{-\mathcal{P}} \\
& =\frac{(n-1) !}{n(2 \pi)^{n}} \int_{\xi, \eta} \Theta \delta\left(\mathbf{t}_{\text {ang }}^{\star}\right) A_{\text {ang }} \mathrm{e}^{-\mathbb{V}(1)}
\end{aligned}
$$

which is an alternative for (2.22) when $\alpha=1$. We may rewrite (3.13) as

$$
p_{n}(1)=p_{n}^{(0)}(1)\left\langle\Theta \delta\left(\mathbf{t}_{\text {ang }}^{*}\right) A_{\text {ang }} \mathrm{e}^{-\mathbb{V}(1)}\right\rangle
$$

where $p_{n}^{(0)}(1)$ and $\mathbb{V}(1)$ are given by the same equations as before, namely (2.26) and (2.23) with $\alpha=1$, and the angular brackets are defined by (2.25). When $\alpha=1$, expression (3.14) is an alternative to (2.24). Both are exact representations of the initial expression for $p_{n}(1)$, but the (3.14) is required if the large- $n$ expansion is to succeed. That will be the subject of section 2.4 .

\subsection{Large- $n$ expansion of $p_{n}(1)$}

\subsubsection{Preliminaries}

We will perform the large- $n$ expansion of the average appearing in (3.14). The expansion of $\mathbb{V}(1)$ is not different from the general case and the result follows directly from (2.36)-(2.39) by setting $\alpha=1$. As before, the indicator $\Theta$ will give corrections that are exponentially small with $n$; we may therefore replace it with unity. The insertion $A_{\text {ang }}$ in (3.14), given explicitly in (3.11), is expanded as

$$
\begin{aligned}
A_{\mathrm{ang}} & =\frac{1}{2} \sum_{m=1}^{n}\left[\gamma_{m}+\beta_{m+1}+\mathcal{O}\left(\gamma_{m}^{3}, \beta_{m+1}^{3}\right)\right] \\
& =\pi+\mathcal{O}\left(n^{-\frac{1}{2}}\right)
\end{aligned}
$$

which, to leading order, is the area of a disk of unit radius. We now investigate the factor $\delta\left(\mathbf{t}_{\text {ang }}^{*}\right)$ for large $n$. In view of (3.12) and (3.4) this requires the asymptotic evaluation of the matrix $\varphi$ defined in (3.5). In the large- $n$ limit the angle differences $\xi_{\ell}=\Phi_{\ell}-\Phi_{\ell-1}$ are independent random variables of average $2 \pi / n$. Hence after setting $\Phi_{0}=0$ and summing $\xi_{\ell}$ on $\ell$ from 1 to

$m$ we have $\Phi_{m}=2 \pi m / n+\mathcal{O}\left(n^{-\frac{1}{2}}\right)$. By doing the sums in (3.5) one then 
finds that $\varphi$ is equal to $\frac{1}{2} n$ times the $2 \times 2$ unit matrix, up to corrections that vanish for $n \rightarrow \infty$. Setting $\hat{R}_{1}=R_{\text {av }} \hat{\rho}_{1}$ we have

$$
\delta\left(\mathbf{t}_{\mathrm{ang}}^{*}\right) \simeq \frac{1}{2} \delta\left(\hat{\rho}_{1}^{\mathrm{c}}\right) \delta\left(\hat{\rho}_{1}^{\mathrm{s}}\right),
$$

where the symbol $\simeq$ indicates validity for $n \rightarrow \infty$. In that limit we can express $\hat{\rho}_{1}$ in terms of the $\hat{X}_{q}$ and $\hat{Y}_{q}$ by means of the relation [18]

$$
\hat{\rho}_{1} \simeq n^{\frac{1}{2}}\left(\hat{X}_{1}-\hat{Y}_{1}\right)
$$

Using (3.15)-(3.17) in (3.14) we find that

$$
p_{n}(1) \simeq p_{n}^{(0)}(1) \times \frac{1}{2} \pi n\left\langle\delta\left(\hat{X}_{1}^{\mathrm{c}}-\hat{Y}_{1}^{\mathrm{c}}\right) \delta\left(\hat{X}_{1}^{\mathrm{s}}-\hat{Y}_{1}^{\mathrm{s}}\right) \mathrm{e}^{-\mathbb{V}_{1}(1)}\right\rangle,
$$

where $\mathbb{V}_{1}(1)$ is given by (2.37) with $\alpha=1$ and $\langle\ldots\rangle$ is the average with respect to the Gaussian weight (2.41).

\subsubsection{The Gaussian integrations}

In (3.18) the integrations on the Fourier variables $\hat{X}_{q}$ and $\hat{Y}_{q}$ with $q \neq \pm 1$ may be carried out in the same way as before and lead to

$$
\begin{aligned}
p_{n}(1) \simeq & p_{n}^{(0)}(1) \times \frac{1}{2} \pi n \prod_{q=2}^{\infty}\left(1-q^{-2}\right)^{-2} \\
& \times\left\langle\delta\left(\hat{X}_{1}^{\mathrm{c}}-\hat{Y}_{1}^{\mathrm{c}}\right) \delta\left(\hat{X}_{1}^{\mathrm{s}}-\hat{Y}_{1}^{\mathrm{s}}\right) \mathrm{e}^{-\hat{X}_{1} \hat{X}_{-1}+\hat{Y}_{1} \hat{Y}_{-1}}\right\rangle,
\end{aligned}
$$

where the terms in the exponential represent the $q= \pm 1$ contribution to $\mathbb{V}_{1}(1)$. The average $\langle\ldots\rangle$ in (3.19) reads explicitly

$$
\langle\ldots\rangle=\frac{1}{2 \pi^{2}} \int \mathrm{d} \hat{X}_{1} \mathrm{~d} \hat{X}_{-1} \mathrm{~d} \hat{Y}_{1} \mathrm{~d} \hat{Y}_{-1} \ldots \mathrm{e}^{-2 \hat{X}_{1} \hat{X}_{-1}-\hat{Y}_{1} \hat{Y}_{-1}}
$$

as follows from (2.41) when restricted to its $q= \pm 1$ Fourier components. When (3.20) and (3.19) are combined, the terms $\hat{Y}_{1} \hat{Y}_{-1}$ in the exponents cancel and the average in (3.19) turns out to be equal to $1 /(3 \pi)$. Without the delta function insertions in the angular brackets, these final integrations on $\hat{Y}_{ \pm 1}$ would have led to a divergence. The fact that they now remain finite confirms the validity of our approach.

After combining everything and still using that $\prod_{q=2}^{\infty}\left(1-q^{-2}\right)=\frac{1}{2}$, we obtain the main result of this section: the sidedness probability $p_{n}(1)$ of the Crofton cell is given by

$$
p_{n}(1) \simeq \frac{2}{3} n p_{n}^{(0)}(1), \quad n \rightarrow \infty
$$

with $p_{n}^{(0)}(1)$ given by (1.2). This is what was announced in section 1.4 of the introduction. 


\section{The typical cell in a Poisson line tessella- tion}

In this section we continue our study of the case $\alpha=1$, that is, the Poisson line tessellation. But whereas in section 3 we studied the Crofton cell (or zerocell), we will now consider the typical cell. The preceding results give rise to a corollary concerning the typical cell that can be derived with little effort. Let $p_{n}^{\text {typ }}(1)$ denote the sidedness probability of the typical cell in the Poisson line tessellation. As was shown in detail by Calka [16], the expression for $p_{n}^{\text {typ }}(1)$ differs from equation (3.8) for $p_{n}(1)$ by the insertion in the integrand of the latter of an extra factor $\langle A\rangle^{\text {typ }} / A\left(\mathbf{R}^{[n]}\right)$, where $\langle A\rangle^{\text {typ }}=1 / \pi \lambda^{2}$ is the average cell area [2] in the Poisson line tessellation.

The extra $1 / A\left(\mathbf{R}^{[n]}\right)$ cancels the $A\left(\mathbf{R}^{[n]}\right)$ present in (3.10) and hence

$$
p_{n}^{\text {typ }}(1)=\frac{\lambda^{n-2}}{\pi n !} \int_{0}^{2 \pi} \mathrm{d} \Phi_{1} \ldots \mathrm{d} \Phi_{n} \int_{0}^{\infty} \mathrm{d} R_{1} \ldots \mathrm{d} R_{n} \delta\left(\mathbf{t}^{*}\left(\mathbf{R}^{[n]}\right)\right) \chi\left(\mathbf{R}^{[n]}\right) \mathrm{e}^{-\mathcal{P}\left(\mathbf{R}^{[n]}\right)} .
$$

In the limit $n \rightarrow \infty$ this is easily evaluated by the methods of sections 3.3 and 3.4. The integral on $R_{\mathrm{av}}$ now has an extra factor $R_{\mathrm{av}}^{-2}$ in its integrand and as a consequence we get instead of (3.14) the expression

$$
p_{n}^{\text {typ }}(1)=\frac{4 \pi}{(n-1)(n-2)}\left\langle\Theta \delta\left(\mathbf{t}_{\mathrm{ang}}^{*}\right)\left(1+n^{-1} V\right)^{-2} \mathrm{e}^{-\mathbb{V}(1)}\right\rangle,
$$

where we have again set $\lambda=1$. Equation (4.2) is still a fully exact expression for the sidedness of the typical cell. ¿From here on, again, we have to resort to a large- $n$ expansion. Since $\left(1+n^{-1} V\right)^{-2}=1+\mathcal{O}\left(n^{-1}\right)$, the average in (4.2) is to leading order in $n$ identical to the one in (3.14) except for the absence of the insertion $A_{\text {ang }}$, which to leading order is equal to $\pi$. Upon combining these considerations we get from (4.2)

$$
p_{n}^{\text {typ }}(1) \simeq \frac{8}{3} n^{-1} p_{n}^{(0)}(1), \quad n \rightarrow \infty .
$$

This is our final result, announced in section 1.4, for the asymptotic sidedness probability of the typical cell. Comparison of (3.21) and (4.3) shows that the zero-cell and the typical cell sidedness probabilities have the ratio

$$
\frac{p_{n}(1)}{p_{n}^{\text {typ }}(1)} \simeq \frac{1}{4} n^{2}=\frac{\pi R_{\mathrm{c}}^{2}}{\langle A\rangle^{\text {typ }}}, \quad n \rightarrow \infty,
$$

where $\pi R_{\mathrm{c}}^{2}=n^{2} / 4 \pi$ [see (2.28) $]$ is the average area of an $n$-sided Crofton cell and $\langle A\rangle^{\text {typ }}=1 / \pi$ is the average typical-cell area. Equation (4.4) is heuristically obvious. 


\section{$5 \quad$ Properties beyond $p_{n}$}

\subsection{General}

In this section we return to a general value of the parameter $\alpha$. The analysis of sections 2 and 3 focused on finding the sidedness probability $p_{n}$. It started from a weight functional for the zero-cell expressed initially [in (2.4)] as $\chi \exp \left(-\mathcal{A}_{\alpha}\right)$. In the course of the analysis we were led to transform this functional into $\exp \left(-\mathbb{V}_{1}\right)$ where $\mathbb{V}_{1}$ is quadratic in the Fourier variables $\hat{X}_{q}$ and $\hat{Y}_{q}$, which in turn are Gaussian distributed according to (2.41). The transformation is valid in the limit $n \rightarrow \infty$ and the resulting weight $\exp \left(-\mathbb{V}_{1}\right)$ may be used to calculate arbitrary averages of functionals of the cell perimeter, provided that sums and products on the wavenumber $q$ converge sufficiently rapidly. We will limit ourselves below to discussing only one of these cell averages.

\section{$5.2 \quad$ Fluctuations of $R_{m}$ around $R_{\text {av }}$}

Important information on the cell shape is contained in the root mean square deviation $\sigma_{R}$ of the vectors $R_{m}$ from their average $R_{\mathrm{av}}$. Straightforward calculation [18] yields for this quantity the expression

$$
\begin{aligned}
\sigma_{R}^{2} & \equiv n^{-1} \sum_{m=1}^{n}\left\langle\left(R_{m}-R_{\mathrm{av}}\right)^{2}\right\rangle \\
& \simeq 2 n^{-1} R_{\mathrm{c}}^{2} \sum_{q=1}^{\infty} q^{-4}\left\langle\left|\hat{X}_{q}-\hat{Y}_{q}\right|^{2}\right\rangle, \quad n \rightarrow \infty
\end{aligned}
$$

It only requires a Gaussian integration to find that for $n \rightarrow \infty$

$$
\left\langle\left|\hat{X}_{q}-\hat{Y}_{q}\right|^{2}\right\rangle \simeq \frac{3}{2} \Lambda_{q}^{-1}(\alpha), \quad q=2,3, \ldots, \quad \alpha \geq 1
$$

A special case is

$$
\left\langle\left|\hat{X}_{1}-\hat{Y}_{1}\right|^{2}\right\rangle \simeq \begin{cases}\frac{3}{2} \Lambda_{1}^{-1}(\alpha), & \alpha>1 \\ 0, & \alpha=1\end{cases}
$$

where the discontinuity at $\alpha=1$ is due to the centering condition that we applied in that case. Substitution in (5.1) of (5.2) and (5.3), as well as of the explicit expression (2.28) for $R_{\mathrm{c}}$, shows that

$$
\sigma_{R} \simeq \begin{cases}c(\alpha) n^{\frac{1}{\alpha}-\frac{1}{2}}, & \alpha>1 \\ \bar{c}(1) n^{\frac{1}{2}}, & \alpha=1\end{cases}
$$


where the coefficients have the properties

$$
\begin{aligned}
& c(\alpha) \simeq \frac{1}{2 \pi(\alpha-1)^{\frac{1}{2}}}, \quad \alpha \rightarrow 1, \\
& \bar{c}(1)=\frac{3}{2 \pi^{2}} \sum_{q=2}^{\infty} \frac{1}{\left(q^{2}-1\right)^{2}}=\frac{1}{16}\left(1-\frac{33}{4 \pi^{2}}\right)=0.010256 \ldots
\end{aligned}
$$

The divergence of $c(\alpha)$ as $\alpha \rightarrow 1$ is due to the divergence of the $q=1$ term in the sum in (5.1); it demonstrates once more the tendency of the center of the cell to become delocalized from the origin in that limit. For $\alpha=1$ we obtain discontinuously the value of $\bar{c}(1)$ due to our centering of the cell.

\section{Conclusion and final remarks}

We have determined the asymptotic large- $n$ expansion of the sidedness probability $p_{n}(\alpha)$ of the zero-cell in a family of tessellations dependent on a parameter $\alpha$. Special cases are the typical Poisson-Voronoi cell $(\alpha=2)$, and the Crofton cell $(\alpha=1)$. The latter turned out to be a singular point in the parameter range and required an extension of previously known methods.

We end with a few remarks about further research to which this work opens the door. The first remark concerns the parameter range $0<\alpha<1$. In this range the density $\rho(\mathbf{R})$ and the probability distribution $P(\mathbf{R})$ defined in (2.3) are still integrable in the origin. However, $\Lambda_{1}(\alpha)$ is negative, which indicates a tendency for the origin to be away from the cell center defined by equation (3.1). Exploring the range $0<\alpha<1$ further would definitely be of interest; it is no longer certain that in that case, too, the zero-cell will tend to a circle in the large- $n$ limit.

Secondly, the analytic method of this work points the way to a new Monte Carlo simulation algorithm for the Crofton cell, similar to the one that was used [20] successfully for the Voronoi cell, and capable of producing accurate $p_{n}$ values for all finite $n$.

Thirdly, one may consider sidedness correlations between distinct cells, whether neighboring or separated by a larger distance. In the Voronoi case $(\alpha=2)$, the correlation between adjacent cells is commonly denoted $m_{n}$ and defined as the average sidedness of a cell given that its neighbor is $n$-sided. To our knowledge, no one has yet attempted to determine the analog of this quantity for the Poisson line tessellation.

We will leave these matters for the future. 


\section{Acknowledgments}

The second author wishes to acknowledge the support of the French ANR Project "mipomodim" No. ANR-05-BLAN-0017.

\section{References}

[1] A. Okabe, B. Boots, K. Sugihara, and S.N. Chiu, Spatial tessellations: concepts and applications of Voronoi diagrams, second edition (John Wiley \& Sons Ltd., Chichester, 2000).

[2] S. Goudsmit, Rev. Mod. Phys. 17, 321 (1945).

[3] R.E. Miles, Proc. Nat. Acad. Sci. USA 52, 901 (1964).

[4] R.E. Miles, Proc. Nat. Acad. Sci. USA 52, 1157 (1964).

[5] R.E. Miles, Advances in Math. 10, 256 (1973).

[6] G. Matheron, Random sets and integral geometry (John Wiley \& Sons Ltd., 1975).

[7] I.N. Kovalenko, J. Appl. Math. Stochastic Anal. 11, 369 (1998).

[8] I.N. Kovalenko, J. Appl. Math. Stochastic Anal. 12, 301 (1999).

[9] A. Goldman, Ann. Probab., 26, 1727 (1998).

[10] D. Hug, M. Reitzner, and R. Schneider, Adv. in Appl. Probab. 36, 667 (2004).

[11] L.A. Santaló, Introduction to integral geometry, Publ. Inst. Math. Univ. Nancago II, Hermann \& Cie, Paris (1953). This formula expresses the length of a planar curve in terms of its number of intersections with the set of straight lines in that plane.

[12] J.C. Tanner, J. Appl. Probab. 20, 400 (1983).

[13] I. Crain and R.E. Miles, J. Stat. Comput. Simul. 4, 293 (1976).

[14] E.I. George, J. Appl. Probab. 24, 557 (1987).

[15] J. Michel and K. Paroux, Methodol. Comput. Appl. Probab. (2007), available online.

[16] P. Calka, Adv. in Appl. Probab. 35, 551 (2003).

[17] H.J. Hilhorst, J. Stat. Mech. L02003 (2005). 
[18] H.J. Hilhorst, J. Stat. Mech. P09005 (2005).

[19] H.J. Hilhorst, Eur. Phys. J. B, available online (2008).

[20] H.J. Hilhorst, J. Phys. A 40, 2615 (2007).

[21] H.J. Hilhorst, J. Phys. A 39, 7227 (2006).

[22] D. Hug and R. Schneider, Geom. Funct. Anal. 17, 156 (2007).

[23] J. Møller, Lectures on random Voronoi tessellations, Springer-Verlag (1994).

[24] R.E. Miles, Adv. in Appl. Probab. 27, 397 (1995).

[25] P. Calka and T. Schreiber, Ann. Probab. 33, 1625 (2005).

[26] R. Schneider, Convex bodies - the Brunn-Minkowski theory (Cambridge University Press, Cambridge, 1993).

[27] R.E. Miles and R.J. Maillardet, J. Appl. Probab. 19 A, 97 (1982).

[28] P. Calka, Adv. in Appl. Probab. 35, 863 (2003).

[29] D.R. Brillinger, Times series data analysis and theory (Holt, Rinehard and Winston, New York, 1975).

[30] D.R. Brillinger and M. Rosenblatt, Spectral analysis of time series, 183 (B. Harris, Wiley, New York, 1967). 